'Unidad de Investigación Epidemiológica y en Servicios de Salud Querétaro, México e Instituto Mexicano del Seguro Social. ${ }^{2}$ Unidad de Medicina Familiar Número 6 San Juan del Río, Querétaro, México.

Recibido el 2 de abril de 2014, aceptado el 30 de marzo de 2015.

Conflictos de intereses: ninguno que declarar.

Correspondencia a: Liliana Galicia Rodríguez Av. 5 de febrero S/N, Esq.

Zaragoza. Col. Centro. C.P. Querétaro de Arteaga. Querétaro. México. 76000.

Teléfono: (442) 2112300

Ext. 51150 y 51113. Liliana.galicia@imss.gob.mx

\section{Costo de la polifarmacia en el paciente con diabetes mellitus tipo 2}

\author{
LUZ MARÍA GARCÍA A. ${ }^{1,2}$, ENRIQUE VILLARREAL R. ${ }^{1}$, \\ LILIANA GALICIA R. ${ }^{1}$, LIDIA MARTÍNEZ G. ${ }^{1}$, \\ EMMA ROSA VARGAS D. ${ }^{1}$
}

\section{The cost of polypharmacy in patients with type 2 diabetes mellitus}

\begin{abstract}
Background: Polypharmacy or the concomitant use of three or more medications, may increase the complexity of health care and its costs. Aim: To determine the costs of polypharmacy in patients with Type 2 Diabetes Mellitus in a Mexican population sample. Patients and Methods: Analysis of health care costs in 257 patients with Type 2 Diabetes Mellitus from two family care facilities, who had at least five consultations during one year. The cost of professional care by family physicians, pharmacological care and medications were considered to calculate the total expenses. The price of medications and the number of units consumed in one year were used to determine pharmacological expenses. Medications were grouped to determine costs derived from complications and concomitant diseases. Costs were calculated in US dollars (USD). Results: The mean cost derived from family physician fees was USD 82.32 and from pharmacy fees USD 29.37. The mean cost of medications for diabetes treatment was USD 33.31, for the management of complications USD 13.9 and for management of concomitant diseases USD 23.7, rendering a total cost of USD 70.92. Thus, the total annual care cost of a diabetic patient was USD 182.61. Conclusions: Medications represent less than $50 \%$ of total expenses of diabetic patients with polypharmacy.
\end{abstract}

(Rev Med Chile 2015; 143: 606-611)

Key words: Diabetes complications; Diabetes Mellitus Type 2; Health care costs; Health economics and organizations; Polypharmacy.

\section{L} a diabetes mellitus tipo 2 (DM2), debido a su asociación con múltiples complicaciones microvasculares y macrovasculares, es considerada como un trastorno crónico complejo, en el cual sólo en etapas tempranas es suficiente la monoterapia, partiendo del concepto fundamental que reafirma que una alimentación adecuada acompañada de actividad física constituye el cimiento del tratamiento. Sin embargo, esto que parece tan sencillo resulta difícil de conseguir en la clínica; en consecuencia se tiene que recurrir al uso de múltiples medicamentos dirigidos a corregir la hiperglucemia ${ }^{1-4}$.

La polifarmacia es definida por la Organización
Mundial de la Salud como el consumo simultáneo de tres o más medicamentos por un mismo paciente, la cual se asocia a una mayor complejidad del manejo terapéutico e incrementa el riesgo de sufrir efectos adversos, de cometer errores en la toma de fármacos, de disminuir la adherencia al tratamiento y de aumentar los costos. Esto constituye hoy un problema, porque en muchos países se desconoce su magnitud, aunado en algunos casos a la prescripción inadecuada del tratamiento, el cual se debe suspender o modificar ${ }^{5-8}$.

El manejo del paciente diabético, por ser una patología crónica, implica consumo constante de recursos y aunado a ellos, el uso de la polifarmacia 
puede incrementar estas necesidades y en una dinámica caracterizada por la economía de la salud, la estimación de los gastos se vuelve una prioridad.

En los sistemas de salud, el uso de evaluaciones económicas ha tomado cada vez más fuerza a nivel mundial, debido a que existe la necesidad de generar una provisión de servicios sanitarios de calidad que contenga una demanda potencialmente ilimitada, en un contexto de recursos escasos y, por tanto, el análisis económico representa un valioso mecanismo que mejora la eficiencia de los procesos de distribución presupuestaria entre los distintos niveles de atención en salud. La economía coloca el acento en la asignación recursos, enfatizando el concepto de costo, que corresponde al valor del consumo de recursos en que se incurre para generar un servicio, el cual puede ser económico o financiero ${ }^{9-11}$.

El objetivo de este trabajo es determinar el costo de la polifarmacia en el paciente con diabetes mellitus tipo 2 .

\section{Pacientes y Método}

Se llevó a cabo un estudio de costos en pacientes con diagnóstico de DM2 con polifarmacia, derechohabientes de dos unidades de medicina familiar del Instituto Mexicano del Seguro Social en San Juan del Río, Querétaro, México, del 1 de septiembre de 2012 al 31 de agosto de 2013.

La polifarmacia se definió como el consumo de tres o más medicamentos por un mismo paciente en un período de por lo menos un $\mathrm{mes}^{5}$.

Se incluyeron pacientes con diagnóstico de DM2 con registro en expediente electrónico, con polifarmacia y que hayan acudido a cinco consultas por lo menos durante un año para el control de dicha enfermedad. Se eliminaron a los pacientes que no contaban con receta elaborada en expediente electrónico o que utilizaran para su control medicamentos adquiridos en forma extrainstitucional.

El estudio se realizó en 257 pacientes, muestra calculada con la fórmula de promedios para población finita, con un nivel de confianza de 95\% para una zona de rechazo $(Z=1,64)$, en una población de 5.000 diabéticos, considerando un costo para los medicamentos utilizados de $\$ 700,00$ anuales, con una desviación estándar de 17,60 y margen de error de 1,76. Los costos en moneda mexicana se convirtieron en dólares estadounidenses (USD), en este trabajo.

La técnica muestral utilizada fue la aleatoria simple por conglomerados, considerando como conglomerado las unidades de medicina familiar y los consultorios. El marco muestral empleado fue el listado de pacientes con DM2 adscritos al área de informática médica y archivo clínico (ARIMAC) y que acudieron a consulta del 1 de septiembre de $2012 \mathrm{al} 31$ de agosto de 2013. Se analizó la cantidad de medicamentos prescritos en la receta médica electrónica, el tiempo de administración indicada y las unidades prescritas de cada uno de ellos, el número de consultas y la atención en farmacia en el último año.

Las variables estudiadas incluyeron características sociodemográficas (edad, sexo, estado civil, escolaridad y ocupación) y entidad patológica (DM2, comorbilidades y complicaciones).

La estimación del costo de la polifarmacia contempló tres dimensiones: consulta médico familiar, atención en farmacia y fármacos. Para la consulta de médico familiar y la atención en farmacia se investigó el uso promedio anual del servicio, el cual se relacionó con el costo unitario para obtener el costo promedio. El costo unitario de estos servicios se estimó con la técnica de tiempos y movimientos identificando para ello los insumos humanos, materiales y de servicios, los cuales se ajustaron por tiempo de producción para identificar el costo del minuto, cantidad que posteriormente se relacionó con la duración de la atención en cada uno de los servicios.

El costo de los medicamentos se estimó identificando el tipo de medicamento, las unidades consumidas al año y el costo por unidad. Estas cantidades al multiplicarse permitieron identificar el costo anual por tipo de medicamento. En un segundo momento se agruparon los medicamentos empleados para el control de la glicemia, los empleados en el manejo de las complicaciones y los empleados en el manejo de las comorbilidades, la suma de todos ellos permitió identificar el costo total en medicamentos.

La recolección de los datos se realizó del expediente electrónico del paciente seleccionado, cuando cumplió con los criterios de selección se analizaron cada una de las consultas que tuvo en el lapso de una año con motivo del control de la DM2, y se registró el número de citas, los medicamentos prescritos en cada visita con la indicación 
de administrarlos por lo menos durante un mes, la cantidad de unidades otorgadas de los mismos y el número de ocasiones que fueron indicados durante el tiempo analizado, y el número de atenciones en la farmacia.

El análisis estadístico incluyó promedios, porcentajes, intervalos de confianza y proyección del costo a diferentes poblaciones de diabéticos.

\section{Resultados}

En la población estudiada, la edad promedio fue 61,47 años (IC 95\%; 60,04-62,89), predominó el sexo femenino con $59,5 \%$ (IC 95\%; 53,5-65,5), el estado civil casado con 71,6\% (IC 95\%; 66,177,1 ), la escolaridad primaria con $32,3 \%$ (IC $95 \%$; 26,6-38,0) y el 50,2\% (IC 95\%; 44,1-56,3) realizaba actividades del hogar. El tiempo de evolución desde el diagnóstico de diabetes mellitus tipo 2 era 11,75 años (IC 95\%; 10,84-12,66), el promedio de consultas anuales fue 7,89 (IC 95\%; 7,69-8,09) y el promedio de medicamentos 6,43 unidades diarias (IC 95\%; 6,15-6,71).

La complicación que se presentó con mayor frecuencia fue la neuropatía en 33,9\% (IC 95\%; $28,1-39,7)$ y la comorbilidad que acompañaba más frecuentemente a la diabetes mellitus era la hipertensión arterial en 51,4\% (IC 95\%; 45,3-57,5).

El costo promedio anual en medicamentos utilizado en el control de la glicemia fue USD 33,31, para el manejo de complicaciones USD 13,90 y de comorbilidades USD 23,70. En la Tabla 1 se presenta el gasto por cada una de las comorbilidades y las complicaciones.

El uso promedio anual en consulta médico familiar y farmacia fue USD 7,89, el costo unitario en medicina familiar se estimó en USD 10,43 y el costo promedio total en USD 82,32, en farmacia el costo unitario se calculó en USD 3,72 y el costo promedio anual fue USD 29,37.

El costo promedio anual de la atención del paciente diabético con polifarmacia fue USD 182,61. La Tabla 2 muestra el costo promedio para cada uno de los insumos requeridos.

La proyección de los costos para los pacientes diabéticos con polifarmacia varía de acuerdo a la población derechohabiente. En una unidad de medicina familiar con 7 consultorios que cuente con una población de 35.000 derechohabientes, prevalencia de $4 \%$ de diabetes y 1.400 pacientes

\section{Tabla 1. Costo promedio anual en medicamentos por tipo de complicación o comorbilidad del paciente diabético en primer nivel de atención}

\begin{tabular}{|lr|}
\hline Entidad patológica & Costo promedio* \\
Patología de base & \\
Diabetes & 33,31 \\
Complicaciones & \\
Insuficiencia renal crónica & 8,65 \\
Neuropatía & 4,69 \\
Retinopatía & 0,38 \\
Pie diabético & 0,18 \\
Subtotal de complicaciones & 13,90 \\
Comorbilidades & \\
Dislipidemia & 5,98 \\
Osteoporosis & 2,82 \\
Enfisema pulmonar & 2,45 \\
Hipertensión arterial & 1,88 \\
Trastorno mixto ansiedad y depresión & 1,79 \\
Glaucoma & 1,71 \\
Hipertrofia prostática benigna & 1,50 \\
Cardiopatía & 1,40 \\
Onicomicosis & 0,98 \\
Osteoartritis & 0,85 \\
Dispepsia & 0,84 \\
Insuficiencia vascular & 0,53 \\
Artritis reumatoide & 0,26 \\
Gastritis & 0,22 \\
Hiperuricemia & 0,15 \\
Asma bronquial & 0,14 \\
Hipotiroidismo & 0,08 \\
Hemorroiditis y fístula anal & 0,06 \\
Epilepsia & 0,03 \\
Vértigo & 0,92 \\
Subtotal de comorbilidades & \\
Total del costo promedio & $1 n$ \\
\hline & \\
\hline libenclamida, Metformina, Acar & \\
\hline & \\
\hline
\end{tabular}

${ }^{1}$ Glibenclamida, Metformina, Acarbosa, Insulina Intermedia, Insulina acción rápida, Insulina Glargina, Insulina Lispro, Pioglitazona, ASA $300 \mathrm{mg} .{ }^{2}$ Alfacetoanalogos, Captopril, Furosemide, Nifedipino 30 mg, Losartan, Felodipino, Amlodipino, Ácido fólico $5 \mathrm{mg}$, Fumarato ferroso. ${ }^{3}$ Complejo b, Imipramina, Gabapentina, Carbamazepina. ${ }^{4}$ Hipromelosa, Latanoprost, Timolol. ${ }^{5}$ Pentoxifilina. ${ }^{6}$ Bezafibrato, Pravastatina, Atorvastatina. ${ }^{7}$ Azatioprina, Raloxifeno, Sulindaco, Celecoxib. ${ }^{8}$ Beclometasona, Budesonida, Salmeterol con fluticasona, Flunarizina, Oxígeno. ${ }^{9}$ Enalapril, Metoprolol, Clortalidona, Hidroclorotiazida, Propranolol $40 \mathrm{mg}$, Nifedipino $10 \mathrm{mg}$, Telmisartán. ${ }^{10}$ Clonazepam gotas, Clonazepam tabs, Diazepam, Citalopram, Paroxetina. ${ }^{11}$ Dorzolamida timolol. ${ }^{12}$ Finasteride, Tamsulosina. ${ }^{13}$ Digoxina, Prazosina, Amiodarona, Verapamilo, Isosorbide 10 mg, Acenocumarina, Clopidogrel, Alfametildopa. ${ }^{14} \mathrm{Keto}-$ conazol, Itraconazol, Miconazol. ${ }^{15}$ Paracetamol, Diclofenaco, Naproxeno, Piroxicam, Calcitriol, Calcio efervesc. ${ }^{16} \mathrm{Al}$ y Mg, Ranitidina, Metoclopramida, Plantago. ${ }^{17}$ Pentoxifilina. ${ }^{18} \mathrm{Me}-$ totrexate, Cloroquina. ${ }^{19} \mathrm{O}$ meprazol, Pinaverio. ${ }^{20} \mathrm{Alopurinol}$. ${ }^{21}$ Salbutamol spray, Ipratropio. ${ }^{22}$ Levotiroxina. ${ }^{23}$ Senosidos, Lidocaina c/hidrocortisona. ${ }^{24}$ Fenitoina, Valproato de magnesio. ${ }^{25}$ Cinarizina. ${ }^{*}$ Costos expresados en dólares estadounidenses. 
Tabla 2. Costo promedio anual por tipo de insumo en el paciente diabético en primer nivel de atención

\begin{tabular}{|lc|}
\hline Tipo de insumo & Costo promedio* \\
\hline Consulta médico familiar & 82,32 \\
\hline Farmacia & 29,37 \\
\hline Medicamento & 70,92 \\
\hline Costo promedio anual & 182,61 \\
\hline
\end{tabular}

*Costos expresados en dólares estadounidenses.

diabéticos, se invierte USD 115,242 en consulta médico familiar, USD 41,121 en farmacia y USD 99,289 en medicamento. Del gasto en medicamentos, USD 39,975 corresponde al tratamiento de la DM2, USD 16,684 para tratar las complicaciones y USD 28,446 para tratar las comorbilidades. El costo total anual de la polifarmacia fue USD 255,652. La Tabla 3 presenta la proyección de costo anual de la polifarmacia en los pacientes diabéticos tipo 2 en primer nivel de atención.

\section{Discusión}

Este trabajo informa sobre el costo de la polifarmacia en el diabético. La diabetes es una enfermedad de evolución crónica que se acompaña de complicaciones micro y macrovasculares, aunando a ellas comorbilidades que requieren de medidas higiénico dietéticas y uso permanente de una amplia gama de fármacos. Este escenario impacta en los recursos económicos que el sistema de salud debe programar y asignar. No obstante, el uso ineficiente parece marcar la pauta, al menos así lo revelan algunos estudios que identifican la falta de apego a la prescripción ${ }^{12}$.

La estimación del costo de la polifarmacia contempló el costo en medicamentos y el costo de la infraestructura necesaria para cumplir con la función de producción. Como se sabe, para dotar de medicamentos a la población se requiere generar la atención médica que se complementa con la atención proporcionada en el servicio de farmacia para el surtimiento de recetas. Esta perspectiva permite identificar de manera precisa la aplicación de los insumos necesarios para generar la atención del paciente diabético con polifarmacia.

Es verdad que no se incluyó el costo de los estudios para evaluar el control y las complicaciones lo cual puede llegar a interpretarse como una limitante, aunque en sentido estricto no la es ya no se contempló como objetivo del trabajo; sin embargo se incluyó el costo de la polifarmacia secundaria a complicaciones, comorbilidades y el control metabólico, lo cual es un acierto del artículo.

Haber identificado el tipo de medicamento y el costo por tipo de entidad nosológica ya sea la patología de base, complicación o comorbilidad es una virtud del trabajo, ya que permite tener una visión más amplia y detallada de la utilización de

Tabla 3. Proyección del costo promedio anual de la polifarmacia en el paciente diabético en primer nivel

\begin{tabular}{|c|c|c|c|c|c|c|c|c|c|}
\hline $\begin{array}{c}n \\
\text { consul- } \\
\text { torios }\end{array}$ & $\begin{array}{l}\text { Población } \\
\text { derecho- } \\
\text { habiente }\end{array}$ & $\begin{array}{c}\text { Pacientes } \\
\text { diabéticos* }\end{array}$ & $\begin{array}{l}\text { Costo de } \\
\text { consulta** }\end{array}$ & $\begin{array}{c}\text { Costo de } \\
\text { farmacia** }\end{array}$ & $\begin{array}{l}\text { Costo de } \\
\text { medica- } \\
\text { mento** }\end{array}$ & $\begin{array}{c}\text { Costo } \\
\text { anual** }\end{array}$ & $\begin{array}{l}\text { Costo me- } \\
\text { dicamento } \\
\text { diabetes** }\end{array}$ & $\begin{array}{l}\text { Costo medica- } \\
\text { mento compli- } \\
\text { caciones }^{* *}\end{array}$ & $\begin{array}{l}\text { Costo medica- } \\
\text { mento comor- } \\
\text { bilidades** }\end{array}$ \\
\hline 1 & 5.000 & 200 & 16,463 & 5,874 & 14,184 & 36,522 & 6,663 & 2,781 & 4,741 \\
\hline 4 & 20.000 & 800 & 65,853 & 23,498 & 56,736 & 146,087 & 26,650 & 11,123 & 18,964 \\
\hline 7 & 35.000 & 1.400 & 115,242 & 41,121 & 99,289 & 255,652 & 39,975 & 16,684 & 28,446 \\
\hline 13 & 65.000 & 2.600 & 214,022 & 76,367 & 184,393 & 474,782 & 86,613 & 36,148 & 61,632 \\
\hline 15 & 75.000 & 3.000 & 246,948 & 88,116 & 212,761 & 547,826 & 99,938 & 41,710 & 71,114 \\
\hline 16 & 80.000 & 3.200 & 263,411 & 93,991 & 226,946 & 584,347 & 106,600 & 44,490 & 75,855 \\
\hline \multirow[t]{2}{*}{20} & 100.000 & 4.000 & 329,264 & 117,488 & 283,682 & 730,434 & 133,250 & 55,613 & 94,819 \\
\hline & 500.000 & 20.000 & $1,646,321$ & 587,441 & $1,418,409$ & $3,652,172$ & 666,252 & 278,064 & 474,094 \\
\hline
\end{tabular}

*Prevalencia del $4 \%$ en relación a la población general. **Costos expresados en dólares estadounidenses. 
los recursos destinados para el manejo del paciente diabético. Otros estudios presentan el costo de la polifarmacia de manera global, sin detallar el gasto correspondiente a cada comorbilidad, lo cual puede considerarse como una limitante que en este estudio ha sido superada ${ }^{13,14}$.

El tiempo de evolución de la población estudiada es similar a lo reportado en otros estudios. Esta comparación se extiende al promedio de consultas recibidas y al promedio de fármacos. La implicación es que el resultado identificado puede extrapolarse a otras poblaciones de pacientes diabéticos y ser de utilidad en la proyección y asignación de recursos para el manejo de la polifarmacia ${ }^{15,16}$.

La presencia de la hipertensión arterial como principal comorbilidad en más de la mitad de la población es un escenario que se presenta en otros estudios ${ }^{17}$. Respecto a los medicamentos empleados por el paciente diabético existen algunos que reportan costos unitarios elevados, más sin embargo, cuando se relacionan con el perfil de uso el costo promedio se diluye en función del bajo uso del medicamento en la población. No obstante, esta es la realidad aplicable a una población de pacientes diabéticos. Tal es el caso del clopidogrel y la insulina glargina.

Si el análisis se sitúa en el costo promedio anual del medicamento la crítica se establece en torno al valor identificado. En realidad pareciera ser muy bajo cuando se compara con los valores publicados en el diario oficial de la federación ${ }^{17}$ para la atención para una atención en consulta externa, no obstante, existen otras publicaciones que se asemejan mucho a los resultados aquí presentados ${ }^{1}$. Al respecto deberá considerarse que el costo estimado en el trabajo se da en una institución que maneja economías de escala y además se caracteriza por ser una empresa con amplio poder de mercado, lo que le permite la adquisición de medicamentos a precios muy bajos.

En este mismo sentido, el análisis del costo de la polifarmacia propio de la patología, las complicaciones y las comorbilidades exacerba, aun más, la discusión al identificar valores en el rango de $\$ 13,90$ a $\$ 27,70$ al año. De lo que se debe estar consciente en este caso es que se habla del costo promedio del medicamento en una población de diabéticos, no del costo individual de un paciente específico, el cual puede ser tan alto o tan bajo como el estado de salud en el que se encuentre. Se podría decir que el costo de un paciente dia- bético con insuficiencia renal crónica ${ }^{18}$ supera en mucho el costo proporcionado aquí; no obstante, se deberá estar cierto que en el estudio presentado se estimó el costo promedio de una población de diabéticos lo cual implica que no toda la población presenta todas las comorbilidades o complicaciones estudiadas.

Como se señaló en Metodología, el costo de la polifarmacia no se limita al gasto en medicamentos, este se extiende al costo de la función de producción en extenso, esto es la atención por el médico y en farmacia. En este caso, lo que se aprecia es que los costos se incrementan, pero es una perspectiva real que debe ser considerada por el tomador de decisiones en el momento de asignar recursos a este tipo de población, no contemplarlo así sería una limitante.

Sin embargo, la crítica puede mantenerse cuando se señala que el costo promedio anual de la polifarmacia en el paciente diabético es cercano a los $\$ 182,61$, en principio pareciera ser una cantidad muy baja, sin embargo, cuando se realizan las proyecciones a una población específica se puede apreciar realmente la magnitud de este costo. $\mathrm{Al}$ menos así lo revela el hecho de destinar 10\% del presupuesto en salud a $4 \%$ de la población (diabetes mellitus), este escenario incita a la cultura del uso eficiente de los recursos.

En conclusión, el costo de la polifarmacia se desglosa para el manejo medicamentoso propio de la enfermedad, para las complicaciones y las comorbilidades; en todos los casos el costo del medicamento se acompaña de la función de producción específica necesaria para dotar al paciente del medicamento.

\section{Referencias}

1. Rodríguez Bolaños R, Reynales Shigematsu L, Jiménez Ruíz J, Juárez Márquez S, Hernández Ávila M. Costos directos de atención médica en pacientes con diabetes mellitus tipo 2 en México: análisis de microcosteo. Rev Panam Salud Pública 2010; 28 (6): 412-20.

2. Zárate A, Basurto L, Saucedo R, Hernández-Valencia M. Guía para seleccionar el tratamiento farmacológico en diabetes 2. Rev Med Inst Mex Seguro Soc 2010; 48 (3): 293-6.

3. Collde Tuero G. Revisión de la medicación en ancianos polimedicados. Aten Primaria 2012; 44: 461-2.

4. Mendoza Acosta S. La polifarmacia como un problema 
de salud en el adulto mayor. Rev Hosp Psiquiátrico de la Habana 2012; 9 (3).

5. Organización Mundial de la Salud. Medicamentos: Uso racional de los medicamentos. 2010. [consultado el 24 de septiembre de 2013]. Disponible en: http://www.who. int/mediacentre/factsheets/fs338/es/

6. Organización Mundial de la Salud. Más salud por el dinero, Capítulo 4. Informe sobre la salud en el mundo. Financiación para la cobertura universal. 2010: 65-94. [consultado el 24 de septiembre de 2013]. Disponible en: http://www.who.intwhr201010_cha04_es.pdf

7. Candela Marroquín E, Mateos Iglesia N, Palomo Cobos L. Adecuación de la prescripción farmacéutica en personas de 65 años o más en centros de salud docentes de Cáceres. Rev Esp Salud Pública 2012; 86: 419-34.

8. Zárate V. Evaluaciones económicas en salud: Conceptos básicos y clasificación. Rev Med Chile 2010; 138 (2): 937.

9. Lenz-Alcayaga R. Análisis de costos en evaluaciones económicas en salud: Aspectos introductorios. Rev Med Chile 2010; 138 (2): 88-92.

10. Saldivia S, Vicente B, Torres F. Medición de resultados en evaluaciones económicas. Rev Med Chile 2010; 138 (2): 79-82.

11. Cabrera de León A, Del Castillo Rodríguez J, Domínguez Coello S, Rodríguez Pérez M, Brito Díaz B, Borges Álamo C, et al. Estilo de vida y adherencia al tratamiento de la población canaria con diabetes mellitus tipo 2. Rev Esp Salud Pública 2009; 83: 567-75.

12. Zhang P, Zhang X, Brown J, Vistien D, Sicree R, Shaw
J, et al. Global healthcare expenditures on diabetes for 2010 and 2030. Diabetes Res Clin Pract 2010; 87: 293 301.

13. Santibáñez-Beltrán S, Villarreal-Ríos E, Galicia-Rodríguez L, Martínez-González L, Vargas-Daza E, Ramos-López J. Costo económico de la polifarmacia en el adulto mayor en el primer nivel de atención. Rev Med Inst Mex Seguro Soc 2013; 51 (2): 192-9.

14. Buitrago Ramírez F. Métodos de medida de la adecuación del tratamiento farmacológico en pacientes pluripatológicos, ancianos o polimedicados. Aten Primaria 2013; 45: 19-20.

15. Vázquez-Martínez J, Mercadillo-Pérez M, Celis-Quintal J. Demanda de atención por diabetes mellitus en el primer nivel de atención. Rev Med Inst Mex Seguro Soc 2011; 49 (2): 225-31.

16. Sabag-Ruiz E, Álvarez-Félix A, Celiz-Zepeda S, Gómez-Alcalá A. Complicaciones crónicas en la diabetes mellitus. Prevalencia en una unidad de medicina familiar. Rev Med Inst Mex Seguro Soc 2006; 44 (5): 415-21.

17. Secretaria de Gobernación. Diario Oficial de la Federación. México. 2014. [consultado el 17 de diciembre de 2014]. Disponible en: http://www.dof.gob.mx/index.php

18. Arredondo A, Rangel R, Icazz E. Costo de intervenciones para pacientes con insuficiencia renal crónica Rev. Saúde Pública [online]. 1998; 32 (3): 255-26. [consultado el 17 de diciembre de 2014]. Disponible en: http:// www.scielosp.org/scielo.php?script=sci_arttext\&pi$\mathrm{d}=$ S0034-89101998000300008. 